\title{
Gender nonconforming youth: current perspectives
}

This article was published in the following Dove Press journal:

Adolescent Health, Medicine and Therapeutics

25 May 2017

Number of times this article has been viewed

\section{Diane Ehrensaft \\ Department of Pediatrics, University of California, San Francisco, CA, USA}

Correspondence: Diane Ehrensaft Department of Pediatrics, University of California, 445 Bellevue Avenue Suite 302 Oakland, CA 94610, USA

Email dehrensaft@earthlink.net
Abstract: Beginning with a case vignette, a discussion follows of the reformulation of theories of gender development taking into consideration the recent upsurge of gender nonconforming and transgender youth presenting for gender services and also in the culture at large. The three predominant models of pediatric gender care are reviewed and critiqued, along with a presentation of the recently developed interdisciplinary model of gender care optimal in the treatment of gender nonconforming youth seeking either puberty blockers or cross-sex hormones.

Keywords: gender nonconforming, transgender, pediatric gender care, puberty blockers, cross-sex hormones

\section{Introduction}

The field of interdisciplinary treatment for gender nonconforming children and youth has not just expanded at an astronomically fast rate; to switch metaphors, it has rather been such as a tsunami, with a swell of children and families seeking support and services and stretching existing gender clinics and programs at their seams. This cohort of young people includes those who do not accept the sex assignment given to them at birth, those who do not accept their culture's expectations and rules about gender roles and gender behaviors, and those who present with a combination of both.

The case of Daniel is presented to launch this review of current perspectives on gender nonconforming youth. Daniel was 19 years old and in his first year of college (note: all identifying information has been changed to preserve confidentiality. In addition, the patient in the case vignette has provided written informed consent for the publication of the anonymized case details). Just a few months earlier he had announced to each of his parents, who were divorced, that he was transgender. For some years before that, he had been living as a girl, assuming that he was either a "butch dyke" or a masculine identified bisexual young woman. His father and stepmother's response was, "Yes, of course, it makes perfect sense. We'll support you in whatever you need". His mother's response was quite different, "God gave you a body, why would you want to go against God's will? I am so ashamed. What will I ever tell my family? I've always supported you, but I can't do this".

Taking a history, Daniel reported that by the end of his sophomore year in high school he discovered that he was transgender. Before that, he never had the language for who he was. Up until second grade, he, then she with the name Daisy, truly believed that when she reached puberty she would simply switch gears, grow a penis, get a beard, 
and become a man. From early childhood she dressed like a boy, insisted on wearing her hair short, and was perceived by all as the neighborhood tomboy. When she learned about the physical changes that accompanied female-menstruating, growing breasts, she responded, by her own report: "Whew, I'm so glad I'll never have to go through that'. When an older youth disabused her of her misconception, informing her that she would receive no exemption and she would never grow to be a man because she was born a girl, she was temporarily devastated, coming to the realization that she was now doomed to walk the plank of female development. For her, this was a horrible thought. When she actually got her period in the sixth grade, she experienced, with trepidation, that her fate had been sealed - "I'm cooked, there's no turning back now".

In middle school, Daisy had her first girlfriend; she confided in her older brother about her new romance, and he promptly issued her a label, "You're a dyke". Except Daisy kept protesting, "I like boys, too". For high school, Daisy chose to go to a boarding school, the prime reason being that she was tired of going back and forth between two houses in her postdivorce family, and just wanted one place to settle into. It was a Catholic all-girls school and she got in trouble for having a romantic relationship with another girl at school. She persisted in dating girls, just not ones from her school, and through her peer connections first learned about the concept of transgender. She surfed the internet, joined chat rooms, and came to discover that "transgender would be me". Her then girlfriend, beginning to recognize who her partner really was, began referring to Daisy as D. and using male pronouns for D. D. never felt happier. But D. kept it a secret for 2 years, waiting out the end of high school and the opportunity to start a new life in college before affirming a male identity publicly. D. chose a liberal arts college far away from home and within weeks came out at school as Daniel. By Thanksgiving break, Daniel was ready to disclose to his parents, and that circles back to the beginning of the story.

After disclosing to his parents, Daniel then wanted hormones to align his body with his male identity, envisioning surgeries, including top and genital surgery, in his future, but not right then. Daniel's story is presented as an opener to highlight the two questions, "What is your gender?" and "What is to be done once discovered?" that underlie all existent adolescent gender care.

Daniel's case is not a unique one. One might even say that it is emblematic of the increasing number of youth who are seeking professional services, along with their parents, to sort out their authentic gender and discover ways to affirm that authenticity. In most Western cultures gender has historically been considered bedrock: one is assigned a sex at birth, either male or female, typically based on external appearance of genitalia, and this assignment determines one's gender for the duration of that individual's life. Upon entrance into the 21 st century, that paradigm of gender bedrock has been hit with a sledge hammer; in its stead, we now have gender as moving boulders, with a sensibility of gender not coming in two boxes, but in infinite varieties, and not necessarily stable over the course of one's lifetime. As this has occurred, providers struggle to keep up with newly emerging theories of gender development and standards of care for the proper care of these youth. Just as an example, the World Professional Association for Transgender Health 7 th Edition of the Standards of Care, ${ }^{1}$ released in 2011, is already outdated and in the process of being revamped, with the section on children and adolescents in particular need of an update. The needed changes come most significantly in the area of social gender transitions for prepubertal youth, minimum ages for medical interventions, particularly puberty blockers and cross-sex hormones, but also surgeries for individuals before reaching the age of majority. Regarding numbers, the cohort of gender nonconforming youth seems to have expanded exponentially in the most recent decade, as reported by gender programs serving these children throughout North America and beyond. ${ }^{2,3}$ In negotiating these phenomenal changes in the gender terrain, four major areas have needed to be addressed: the necessity of relearning gender so that health professionals can retool themselves to best serve this group of youth; the tensions between the three models of care; the importance of interdisciplinary collaboration in care; the introduction of medical interventions in the care of the youth.

\section{Reformulate theories of gender development in light of gender nonconforming youth}

Most professionals in the field of gender care have had to unlearn everything taught in training about gender and relearn a new model of gender development. To review the traditional model, children at birth are assigned a sex, male or female, typically based on appearance of external genitalia. If the genitalia were ambiguous in appearance, genital surgical procedures to establish a stable singular sex assignment with matching gender were to be performed as soon as possible, and no later than 18 months. The reasoning behind this, as propounded by Dr John Money and his associates, ${ }^{4}$ was that after $18-24$ months a child is firm in a core gender identity - I am male, I am female, and thereafter it becomes very difficult to change that identity as it is 
already cognitively fixed. Once knowing one's gender label, which is both facilitated and mediated by parents' conscious and unconscious messages and reflections, a child's next developmental task is to learn how to "do" gender. Known as gender role socialization, this process is done in close relationship to one's mother and father, with the underlying assumption that all children will have both. ${ }^{5,6}$ Within the psychoanalytic paradigm, during this same period a tumultuous drama unfolds, the Oedipal phase - children have intense erotic fantasies about their parents: boys will want to marry their mothers, girls their fathers. Through successful negotiation of these fantasies, facilitated by parents' empathy and boundary setting, children will emerge from the Oedipal phase relinquishing those infantile incestuous desires, firming their own heterosexual identities as they forestall gratification and await an opposite sex partner of their own when they reach adulthood. ${ }^{7}$ Within that process they will establish a firm gender identity with a new understanding that one is and always will remain the sex listed on one's birth certificate or assigned early in life (for intersex children). ${ }^{8}$ Throughout middle childhood youth will continue to internalize the gender norms of their culture, and learn to conform to them. With the advent of puberty and the entrance into adolescence, a new phase of gender consolidation occurs as youth awaken to their adult sexual urges and prepare for their gender-divided roles as men or women.

Within the traditional model of gender development, if this developmental trajectory takes a course other than that described above, there is cause for concern for the child, along with scrutiny of the parents, as parents are held accountable for the child's anomalies. To quote Robert Stoller, a pioneer in the treatment of gender disorders in youth in the 20th century, ${ }^{9}$ speaking of "primary transsexual" boys (those nonintersex boys who have been feminine from the first year of life): "As an infant, such a boy usually has an excessively intimate, blissful, skin-to-skin closeness with his mother. This, unfortunately, is not interrupted by his father, a passive distant man who plays no significant part in bringing up his son" (p. 16). In family situations like the one inscribed above by Stoller, professional help was recommended to cure the youth's gender anomalies and to treat the parents so they cease veering their child's gender development in wrong directions because of their own internal conflicts.

For a theory of development to be robust, it should be evident in empirical observation or investigation. The traditional theory of gender development and disordered gender, which is still in use by many, fails that test, for the following reasons: ${ }^{10}$
- Many individuals continue renegotiating their gender throughout childhood or adulthood, with no observable detriment to their mental health;

- Youth may establish a gender identity in concordance with their assigned sex, be firm in that identity, yet not embrace a heterosexual identity, with no aspersion on their emotional well-being. Gender development and sexual identity development are two separate developmental tracks, albeit crossing at certain points.

- Whereas core gender identity is typically concordant with assigned sex based on observable external genitalia, for a minority of people this is not the case, with increasing evidence that gender identity lies not between our legs, in our genitalia and primary sex characteristics, but in our brains and minds. ${ }^{11}$

- Therefore, one's assigned sex at birth may differ from one's core gender identity, not because of poor parental handling or infantile confusions, but because of brain and mind gender messages overriding signals from genitalia, chromosomes, or parental expectations. Recently, this phenomenon of mind over matter has been referred to as "neurological sex", defined as a uniform standard of legal sex based on gender identity, in which brain messages are privileged over anatomy and chromosomes in determining an individual's authentic gender. ${ }^{12}$

In contemporary versions of gender development theory that take into account gender variations as a normal part of the human condition, the understanding is that the sex assigned at birth may match the gender a youth will eventually know themselves to be, but it might not. Each child is presented with a developmental task of weaving together threads of nature, nurture, and culture to establish their individual and unique authentic gender self. This self will be composed of both gender identity - who I know myself to be as male, female, or other, and gender expressions - how I choose to perform my gender, including clothing choices, activity preferences, friendship choices, and so forth. Recently, this transactional relationship between nature, nurture, and culture in gender development has been referred to as the gender web, ${ }^{13}$ broken up into components that consist of the items in Table 1.

In this contemporary model of gender development, added to the three dimensions of nature, nurture, and culture is the fourth dimension: time. Each child alters their gender web as they weave together nature, nurture, and culture, "over time". In other words, gender is neither fixed by age 6 , as in the traditional model, nor static throughout all stages of child and adult development, thus explaining how an individual 
Table I Gender development: elements of the gender web

- Chromosomes

- Hormones

- Hormone receptors

- Gonads/primary sex characteristics

- Secondary sex characteristics

- Brain

- Mind

- Socialization: family, school, religious institutions, community

- Culture: values, ethics, laws, theories, and practices

at age 40 or 50 could come to the realization that the gender they had identified as being is no longer a good fit. It is also recognized that gender development is a discrete and separate track from development of one's sexual identity, and typically proceeds it in a youth's development.

In this model the role of parents and socialization agents is not to shape or reinforce a child's gender identity or expressions, but rather to facilitate it, mirroring back to the child the messages that the child communicates about their preferred gender expressions and articulated gender identity, which may or may not be in concordance with the sex assigned to the child at birth. With the advent of adolescence, it is recognized that some youth's gender trajectories may benefit from medical interventions, including puberty blockers (gonadotropin-releasing hormone [GnRh] agonist) and crosssex hormones to bring the youth's body in better alignment with their affirmed gender identity. ${ }^{14}$ To that end, the model of care that extends from this contemporary theory of gender development is one that strongly relies on interdisciplinary care, especially between mental health and medical providers as they address the holistic medical and psychosocial needs of the emergent cohort of gender nonconforming youth from the perspective of both their psychological and physical development.

\section{Major mental health treatment models for gender nonconforming children and youth}

As of the second decade of the 21 st century, three major treatment models are available for addressing the needs of gender nonconforming children and their families, with overlapping premises based on the contemporary model of gender development outlined above but with distinct differences between them. The first model, represented in the work of Drs Susan Bradley and Ken Zucker, assumes that young children have malleable gender brains, so to speak, and that treatment goals can include helping a young child accept the gender that matches the sex assigned to them at birth. The second model, represented in the work of practitioners in the Netherlands, allows that a child may have knowledge of their gender identity at a young age, but should wait until the advent of adolescence before engaging in any full transition from one gender to another. The third model, represented in the work of an international consortium of gender affirmative theoreticians and practitioners, allows that a child of any age may be cognizant of their authentic identity and will benefit from a social transition at any stage of development. To situate and compare each of the three models, a typical referral that may come the way of a gender specialist, regardless of their orientation, is presented, with the assumption that this potential patient may be in need of services from a young age through adolescence:

Hi Dr, I came across your information while I was researching for my son.

He recently just turned 4 and wants to be a girl and is only drawn to girl toys/clothes for the past 2 years.

We have not spoken with a professional doctor. But wanted to reach out early and find ways we as parents can support him.

Please let me know if you could help.

Thank you!

Dialing back a generation, if this child's name was Kyle and the same query came to a mental health professional participating in, for example, Dr Richard Green's clinic at the University of California Los Angeles, the treatment recommended and then implemented could very well have looked like this:

When he was five, Kyle entered a behavior modification program. [...] Kyle received blue tokens for "desirable" behaviors [...] red ones for "undesirable" behaviors [...]. Blue tokens were redeemable for treats [...]. Red tokens resulted in a loss of blue tokens, periods of isolation, or spanking by father. ${ }^{15}$

Setting a precedent for other clinicians of the time treating children who presented as gender nonconforming, Kyle's treatment at the UCLA program is emblematic of the model implemented during this era, with the goal of helping children accept the sex assigned to them at birth and adopt the culturally defined appropriate gender behaviors that would match that sex assignment, in alignment with the traditional model of gender development. Underlying the treatment was the intent of warding off a homosexual outcome for young effeminate boys. It should be mentioned 
that this model is still practiced today, referred to by some as the reparative model.

Focusing now on contemporary approaches that stand in contrast to the above mode, all of which are to be differentiated from the UCLA program, the three major models, outlined earlier, are typically referred to, in order of presentation, as the following:

- The "live in your own skin" model

- The watchful waiting model

- The gender affirmative model

Below is a review of the manner in which each of these models would approach the treatment of a child or youth who is presenting as gender nonconforming, in their gender identity, gender expressions, or both.

\section{The "live in your own skin" model}

As mentioned earlier, this model was developed by Drs Susan Bradley and Ken Zucker at the Center for Alcoholism and Mental Health gender clinic in Toronto. ${ }^{16}$ The treatment goal of facilitating a young child accepting the gender identity matching the sex assigned to that child at birth, based on the supposition that younger children, in contrast to older youth, have a malleable gender brain, is tied to a medical-social rationale. Specifically, being transgender is a harder way to live one's life, both because of social stigma and potential requested hormonal treatments and surgeries to align a youth's body with their transgender identity. Given the perceived plasticity of the young child's gender brain, best practice would be to introduce interventions to help a child accept the sex assigned to them at birth as their gender identity, with no harm done and indeed added benefit to their psychological and social well-being. As explained by Dr Zucker, employing this strategy results in lowering the odds that "as such a kid gets older, he or she will move into adolescence feeling so uncomfortable about their gender identity that they think that it would be better to live as the other gender and require treatment with hormones and sex reassignment surgery". ${ }^{17}$ In addition to presuming gender identity malleability in young children, the model also assumes that parents' own conflicts or issues about gender likely contribute to a young child's gender dysphoria. With the parents' consent, the "live in your own skin" model employs a combination of behavior modification, ecological interventions, and family system restructuring to facilitate the child arriving at a place of accepting the gender matching their sex assigned at birth. Practices could include taking away cross-gender toys at home and replacing them with "gender-appropriate" toys, altering children's playmate choices to include more same-sex contacts, enrolling the children in "genderappropriate" activities, encouraging the like-sex parent to become more actively involved and the opposite-sex parent to step back in relationship to the child, and offering psychotherapy to both the child and parents. The aim of treatment of the child is to explore the child's gender and solidify a "live in your own skin" outcome, and the treatment with the parents is aimed at investigating conflicts or psychological issues stemming from or contributing to the child's gender dysphoria. If by the arrival of puberty a child is still exhibiting cross-gender identifications and expressing a cross-gender identity, that child should be supported in transitioning to the affirmed gender, including receiving puberty blockers and hormones, once it is assessed through clinical interviews and psychometric testing that the affirmed gender identity is authentic. The reasoning behind this shift in adolescence is as follows: 1) by adolescence it is too late to intervene in facilitating a child living in their own skin, as the sensitive period of malleable brain development of gender has closed; 2) this individual can now be reliably identified as one of the small minority of youth who persist with a cross-gender identity from early childhood into adolescence, an indicator that this identification will most likely remain stable into adulthood. In the live in your own skin model, the parent reaching out for support of her 4-year-old son might be encouraged to engage in the treatment program outlined above, with the goal of helping her child accept that he is a boy, not a girl and with the intent of warding off a transgender outcome.

\section{The watchful waiting model}

The "watchful waiting" model was designed by the members of the interdisciplinary team at the Amsterdam Center of Expertise on Gender Dysphoria, VU University Medical Center, under the leadership of Dr Peggy Cohen-Kettenis. Borrowing from the medical use of GnRH agonists for children exhibiting precocious puberty, the Netherlands team is responsible for introducing the use of puberty blockers for gender purposes, to put a pause on pubertal growth and allow more time for a youth to explore their gender and consolidate their adolescent gender identity, with the future possibility of cross-sex hormone therapy to align their bodies with their affirmed gender identity. In contrast to the live in your own skin approach, a young child's demonstration of gender nonconformity, be it in identity, expressions, or both, is not to be manipulated in 
any way, but observed over time. If a child's cross-gender identifications and affirmations are persistent over time, interventions are made available for a child to consolidate a transgender identity, once it is assessed, through therapeutic intervention and psychometric assessment, as in the best interests of the child. These interventions include social transitions (the shift from one gender to another, including possible name change, gender marker change, and gender pronoun changes), puberty blockers, and later hormones and possible gender-affirming surgeries. No attempts are made to alter a child's gender identity or expressions; yet it is postulated in this model that it would be better to hold off until puberty on any social transitions of a child from one gender to another, and instead give them safe spaces to fully express their gender as they prefer before facilitating any full gender transitions. ${ }^{18,19}$ The rationale for holding off on any social transitions until adolescence is not to ward off a transgender identity but rather that 1 ) it would be advantageous that a child experiences the first stages of physical puberty for that child to best make a determination of the gender that feels most authentic to him/her; 2) given developmental stages of childhood, facilitating a social transition from one gender to another at a young age may create a form of cognitive constriction - the child may be prematurely blocked from considering any other possibilities once moved into a cross-gender status and socially constricted from further childhood gender exploration because now they know the cross-gender identity is what everyone has come to expect from them; 3 ) socially transitioning a child at a very young age may preclude the child from maintaining a realistic understanding of their body and historical status - as a penis-bodied (once a boy) or a vagina-bodied (once a girl) person. In informing their practices, this model, like the live in your own skin model, relies on the data gathered about "persisters" and "desisters", both at their own clinic in the Netherlands and in other international studies, particularly those conducted at the Centre for Addiction and Mental Health (CAMH) gender program in Toronto. In the most recent review of these studies, it was found that $63 \%$ of the children seeking services at a gender clinic at a young age, and diagnosed with gender dysphoria, no longer had that diagnosis at puberty, while $37 \%$ did have the diagnosis consistently from early childhood to adolescence. ${ }^{20}$ Since a large majority of gender nonconforming young children seeking services at gender clinics desist in their gender dysphoria by adolescence, best practices would be to wait and see if the child persists into adolescence before making any significant changes in a child's gender identity.
During the preadolescent waiting period, the children are followed carefully by the clinical team in the watchful waiting model, with the support of outside therapists in the community (which is required before a child can receive medical services), to assure that the children are growing well and getting their emotional needs met, and in preparation for later transitioning and medical interventions if the child proves to be a good candidate. Like in the live in your own skin model, the children going through the program also receive a full battery of psychological tests, documenting not only their gender status but also their cognitive-social-emotional functioning. Some of these instruments are delivered to the children directly, some to their parents or teachers.

If the mother asking for help with her 4-year-old were to attend the Amsterdam clinic with her child, the team might do an assessment and advise that the 4-year-old be followed over time, with the understanding that if her son's declarations of wanting to be a girl persisted over time and if he continued to be drawn only to "girl" toys and activities, consideration of puberty blockers to buy more time to explore gender could certainly happen later, but for now it would be best to let her son continue to be a son free to explore whatever activities he enjoyed, with no corrections on his expressed desire to be a girl.

\section{The gender affirmative model}

The third model of care, the gender affirmative model, is closely aligned with the watchful waiting model but in opposition to the live in your own skin model. Where the gender affirmative model parts ways with the watchful waiting model is in the waiting part.

The gender affirmative model is defined as a method of therapeutic care that includes allowing children to speak for themselves about their self-experienced gender identity and expressions and providing support for them to evolve into their authentic gender selves, no matter at what age. Interventions include social transition from one gender to another and/or evolving gender nonconforming expressions and presentations, as well as later gender-affirming medical interventions (puberty blockers, cross-sex hormones, surgeries). A particular set of premises informs the model, as listed in Table 2.

The model is informed by the contemporary theory of gender development outlined above, with a recognition that although gender evolves over the course of a lifetime, gender identity appears to be a relatively more stable and consistent construct compared to gender expressions. Gender health is defined as a youth's opportunity to live in the gender that feels most real and/or comfortable, or, alternatively, a youth's 
Table 2 Basic premises of the gender affirmative model

- Gender variations are not disorders.

- Gender presentations are diverse and varied across cultures, requiring cultural sensitivity.

- Gender involves an interweaving, over time, of biology; development and socialization; and culture and context.

- Gender may be fluid; it is not always binary.

- If present, individual psychological/psychiatric problems are more often than not secondary to negative interpersonal and cultural reactions to a child.

- Gender pathology lies more in the culture than in the child.

ability to express gender with freedom from restriction, aspersion, or rejection. ${ }^{21}$ When considering a child's gender status, attention is paid to both gender identity and gender expressions, with the understanding that a child's gender identity may communicate something very different about the child than a child's gender expressions might.

Therapeutic goals in the gender affirmative model include:

- Facilitating an authentic gender self

- Alleviating gender stress or distress

- Building gender resilience

- Securing social supports

In contrast to the first two models, no assumption is made that every child exhibiting a gender nonconforming presentation is in need of mental health treatment. Because of the emphasis on social factors affecting the youth, interventions may be targeted at the surrounding environment, rather than the child's individual psyche. This might include interfacing with schools, social and religious institutions, and policymaking bodies to remove the "social" pathology impinging on the child, such as transphobic attitudes and responses, gender policing, or bullying and harassment. Relatedly, parent consultations often take precedence over individual treatment of the child, ${ }^{22-24}$ with provision of services to help a parent make sense of their child's gender nonconformity, work through any extant conflicts and anxieties about their child's gender, and move toward acceptance of their child.

Individual treatment for the child is indicated for one of five reasons: 1) to assess a child's gender status; 2) to afford the child a "room of their own" to explore their gender; 3 ) to identify and attend to any co-occurring psychological issues; 4) to address and ameliorate a child's gender stress or distress; 5) to provide sustenance in the face of a nonaccepting or rejecting social milieu, which might include family, school, religious institution, or community. Some professionals working in this model will call on psychometric or projective measures to gather information about the child; others will rely on observation, play, interviewing, and dialog. If assessment instruments are employed, every effort is made to use protocols that do not rely on binary measures of gender (e.g., Are you a boy or a girl?) and are not pathology oriented, but instead assess strengths as well as weaknesses and differentiate between gender expressions and gender identity.

The basic therapeutic tenet of the gender affirmative model is quite simple: When it comes to knowing a child's gender, it is not for us to tell, but for the children to say. In contrast to the watchful waiting model, once information is gathered to assess a child's gender status, action is taken to allow that child to exercise that gender. Therefore, if after careful consideration, it becomes clear that a young child is affirmed in their gender, demonstrating that the gender they know themselves is different than or opposite to the gender that would match the sex assigned to them at birth, the gender affirmative model supports a social transition to allow that child to fully live in that gender, whether that child is 3,7 , or 17 years old. Such decision-making is governed by stages, rather than ages, both for social transitions and later for medical interventions. Once the child's gender comes into clear focus, which is posited as happening with a child of any age, no need is seen to hold off until adolescence to affirm that gender. This viewpoint is informed by data indicating the psychological harm that can be done, including heightened risk for generalized anxiety, social anxiety, oppositional behaviors, depression, compromised school performance, if a youth experiences themselves living in a gender that is inauthentic to them. ${ }^{25}$

In the gender affirmative model, the mother of the 4-yearold querying about her son's cross-gender interests would be invited in to the consultation room, along with any other parenting figure involved, to report more about what she had been observing in her child's behaviors from infancy to the present; to determine whether her son is showing any signs of stress or distress about his interest in all things girly things; to explore whether her child is indicating cross-gender expressions vs identity. If there was evidence of stress or distress, by parents' report, or if the parents desired to get a clearer picture of their child's gender status, the family would be invited to bring their son in for observation and play sessions. There would then be the opportunity to reflect, in collaboration with the parents or caregivers, on any evidence that this child was consistent in cross-gender declarations, as in "I'm a girl, not a boy", and that these declarations were persistent over time and not attributable to any other problems in life. If that evidence made clear that this child was communicating about a cross-gender identity rather than desired cross- 
gender expressions, and if the parents were supportive of their child's gender identity affirmations, it would not be found necessary to recommend to this mother that she wait until puberty to take action regarding her child's gender identity. Instead, a present social transition to the gender that was more authentic for this child, in this case, female, would be considered. If, on the other hand, the child was happy as he was, if given the latitude to play with whatever he wanted and wear whatever he desired, as a boy, the recommendation to the mother might be to give her son the opportunity to express his gender freely, with the opportunity to return for services as requested. Along with this recommendation would be a reminder that all that can be known is the cross section of this child's gender as he presents it at age 4, a gender that may evolve into another configuration later in childhood, at which point a new assessment may be in order.

\section{Critique of the three models}

In brief, the live in your own skin model has been challenged as causing potential harm to gender nonconforming youth. A Canadian study conducted by Wallace and Russell assessed that in the living-in-your-own-skin model "there appears to be an enhanced risk of fostering proneness to shame, a shamebased identity and vulnerability to depression." ${ }^{26}$ Major health organizations, including the World Professional Association for Transgender Health, the American Psychological Association, and the American Psychiatric Association, have issued statements stipulating that mental health professionals are not to engage in practices that attempt to alter the gender expressions or identity of an individual, including children and adolescents. The watchful waiting model is a highly respected model of care worldwide, offering careful and cautious procedures; but it has run into a snag: many contemporary families in the Netherlands are not content to hold their children back from social transitions until puberty, and have, through both local and international support networks of parents and professionals, proceeded to facilitate their children's social transitions without awaiting clinical approval or waiting until puberty arrives. Parents do this not because they dismiss professional care, but because evidence is accruing that young children thrive when given permission to live in the gender that is most authentic, ${ }^{27,28}$ and are at risk for symptomatic behaviors if prevented from doing so. At the same time, the watchful waiting model is effective in its thorough attention and assessment of the child over time, integrating the services of mental health and medical professionals.

The gender affirmative model is questioned by some on the basis of the lack of evidence-based data that indicates that young children can reliably communicate and have selfknowledge of a transgender identity or benefit from a social transition. There is also concern that the model of listening to the children puts too much weight on a child's self-report. This is a valid concern, and to address it the self-report is embedded within a collaborative model with the child as subject and the collaborative team including the child, parents, and professionals. Together, the team will be making informed determinations about the most appropriate gender pathways to promote a child's gender health, be it a gender social transition, expanded opportunity to express gender in ways that feel authentic to the child, or deeper exploration of underlying issues that may be presenting as gender stress or distress. Such determinations typically involve extensive consultation and observation, but with no requirement for ongoing psychotherapy or psychometric testing, in comparison to the other two models.

\section{Integration of medical and mental health care in adolescence}

All of the three models of care referenced earlier share in common the administration of hormonal treatment in adolescence. The first category would be consideration of $\mathrm{GnRH}$ agonists (puberty blockers) to put a temporary pause on puberty, providing a youth with additional time to explore gender or, alternatively, warding off an unwanted puberty. The latter is particularly true for youth who socially transitioned early in life, living consistently in their affirmed gender from a young age; in those instances administration of puberty blockers could be considered a form of continuity of care, from social transitions to hormonal intervention. The second category includes feminizing or masculinizing hormones to bring a youth's body in better alignment with their affirmed gender identity. The minimal age for being eligible for such treatments may vary among approaches and indeed among clinics adopting the same approach, but there is common agreement that these treatments are in the best interests of the child who has a documented transgender identity. ${ }^{29}$ It should be noted that there is probably no other aspect of adolescent care in which the medical and mental health professionals are so vitally interdependent in both assessment and treatment of the youth. ${ }^{30}$ The reason for this is that each of the interventions has vital interconnected psychological and medical components, requiring an integration of medical evaluation and mental health assessment both to determine appropriateness, assess any medical or psychological impediments to treatment, and monitor follow-up, in terms of effects and supports over time as the youth is administered either the puberty blockers or hormones. 
The role of the medical professional is first to assess the youth's level of puberty development, with an assessment of physical readiness for considerations for puberty blockers, which can be administered as soon as the youth enters Tanner Stage 2 of puberty. The medical professional will be responsible for ordering the lab work and bone density scans necessary to monitor a youth's progress and also to screen for any medical counter-indications to administering the blockers. As RnGH agonists are a completely reversible procedure regarding development of secondary sex characteristics, the medical provider will not need to worry about untoward permanent effects in that regard if the youth decides to go off blockers and return to the unfolding of a physical puberty in concordance with the sex assigned at birth. It should be noted, however, that the provider will need to alert the child and family about any side- or long-term effects of RnGH agonists, including effects on bone mineral density and overall bone health. If, on the other hand, the youth decides to proceed with cross-sex hormones to affirm a gender identity not in concordance with the sex assigned at birth, the medical provider will then be faced with the task of determining if the youth is a good candidate for this next step of treatment. Some youth will have already gone through full puberty before discovering or communicating to others a transgender identity, and the medical provider will be faced with the same task with these youth, with the added feature of explaining to the youth that certain of the developed features of the puberty they have already gone through will not disappear as they go through a second puberty on crosssex hormones. In either case, cross-sex hormones involve a weightier decision than puberty blockers, as these interventions are only partially reversible in terms of secondary sex characteristics, so the provider will want to be cautious and judicious in determining if cross-sex hormones are appropriate for a particular youth.

This is where the mental health professional enters. In all of the models of gender care, the mental health professional is asked to weigh in as to 1) the authentic gender identity of the youth or level of gender dysphoria exhibited by the youth; 2 ) the youth's level of maturity and ability to assent to and follow through on the recommended hormonal treatment; 3 ) the evidence of any coexisting psychological conditions that might interfere with the hormone treatment or that alternatively might bear no weight on the requested treatments or even be alleviated by the hormonal interventions; and 4) the level of family support and willingness to consent to the treatment. In consultation with the medical professional, a decision will be made as to whether a youth is a good candidate for either puberty blockers or cross-sex hormones.
Another critical task for the medical-mental health team is the necessary discussion of fertility implications for each of these interventions. Although advances are being made in reproductive medicine to preserve immature gametes or reproductive tissues for later reproduction, at this point in history a child who begins puberty blockers at Tanner Stage 2 and proceeds directly to cross-sex hormones will be rendered infertile. Administration of testosterone or estrogen to a postpubertal adolescent may compromise a youth's later fertility, or might require going off the hormones for a period of time if a transgender youth who has not had gonad or genital surgeries later in life desires to have a genetically related child. Alternatively, a youth can bank gametes for the future before going on a course of cross-sex hormones, which is a medical possibility but also a psychological challenge for many transgender youth who find this antithetical to their affirmed gender status, requiring a transgender female to attend a fertility clinic and masturbate or a transgender male to undergo a gynecological vaginal ultrasound. Exploring fertility issues before making decisions about blockers or hormones are necessary but sensitive discussions to be had with both the youth and parents, and are best done with the presence of both a medical and a mental health professional who together can provide medical and psychological counsel to the family in this decision affecting later family-building. ${ }^{31}$

Not only is there no other aspect of adolescent care where the teamwork between medical and mental health provider is critical; there is no other domain of youth services in which a mental health provider is so actively involved in medical decision making. Where this has surfaced most recently is in the recent emergence of youth in gender clinics who present as neither male nor female, but rather gender nonbinary or "in the middle", adopting the platform of the multiplicity of gender. The challenge is when these youth ask for a particular medical intervention that achieves that goal of a middle ground - perhaps a touch of testosterone, or chest surgery with no other intervention and a chosen pronoun of "they" rather than "he" or "she". These are new horizons for both medical and mental health professionals today, and there is a mutuality, therefore, in the medical professional training the mental health professional while the mental health professional is in turn training the medical professional in order to integrate the biopsychosocial aspects of care to include the gamut of all the gender nonconforming youth presenting for care. ${ }^{32}$

With that said, it has proved to be critical that mental health professionals involved in this team work be trained gender specialists, with a basic understanding of the medical interventions involved in transgender care, expertise in 
assessing gender dysphoria and identifying a youth's gender identity, and recognition of psychological issues other than gender that might drive a youth's request for a hormonal treatment. For example, a nurse practitioner on a gender team had administered a puberty blocker implant, Supprelin, which could stay in place for a year, after receiving a letter of support from a trained mental health expert recommending such treatment for this youth who presented as gender dysphoric and in need of further exploration of his gender before going forward with puberty. Over the course of the following year, he failed to return for follow up visits. A year had gone by and it was now time to replace the implant, which the nurse practitioner was prepared to do. The mental health member of the team first did a follow-up evaluation of the youth and discovered that he had made no efforts to explore his gender any further, with his motivation to continue on blockers driven by a desire to remain prepubertal for as long as possible. With the psychologist's guidance, the medical provider was able to recognize that the medical intervention as it stood was inappropriate for this youth. The interdisciplinary team informed the youth that he would be able to receive a new implant only if he was simultaneously working with a mental health gender specialist to further explore his gender identity. If that condition was met, once the twelve additional months on the puberty blockers was completed, the youth would then have to make a determination of which puberty path he would take - cross-sex hormones or the unfolding of his male, testosterone-producing puberty.

\section{Conclusion}

In the course of only two decades, sophisticated models for the care of gender nonconforming and transgender youth have evolved. There is an urgent need to provide more research data documenting the efficacy of these different programs, but the recent findings of the Amsterdam group provide hope that the care, particularly within the watchful waiting and gender affirmative models, is promoting gender health. In the Dutch authors' words, the treatment, including puberty suppression, cross-sex hormones, and then in adulthood gender affirmation surgery, "leads to improved psychological functioning of transgender adolescents. While enabling them to make important age-appropriate developmental transitions, it contributes to a satisfactory objective and subjective wellbeing in young adulthood". ${ }^{33}$ The authors propose that not only early medical intervention, but also a comprehensive multidisciplinary approach contributes to the youth's gender health. Reflecting back on Daniel, the youth introduced at the opening of this review, the ability of professionals to aid youth such as Daniel in getting his authentic gender into focus and providing the appropriate treatments to bring that gender in alignment with his body is the key to overall well-being for all youth seeking professional gender care.

\section{Disclosure}

The author reports no conflicts of interest in this work.

\section{References}

1. World Professional Association for Transgender Health. Standards of Care for the Health of Transsexual, Transgender, and Gender Nonconforming People. WPATH; 2011.

2. Hidalgo MA, Ehrensaft D, Tishelman AC, et al. The Gender affirmative model: what we know and what we aim to learn. Hum Dev. 2013;56:285-290

3. Zucker KJ, Wood H, VanderLaan, DP. Models of psycho-pathology in children and adolescents with gender dysphoria. In: Kreukels BPC, T. Steensma TD, de Vries ALC, editors. Gender Dysphoria and Disorders of Sex Development: Progress in Care and Knowledge. New York, NY: Springer; 2014:171-192.

4. Money J, Ehrhardt A. Man \& Woman, Boy \& Girl: Gender Identity from Conception to Maturity. Northvale, NJ: Jason Aronson; 1996.

5. Diamond M. Sex, gender, and identity over the years: a changing perspective. Child Adolesc Psychiatr Clin N Am. 2004;13(3):591-607.

6. Tyson P. A Developmental line of gender identity, gender role, and choice of love object. J Am Psychoanal Assoc. 1982;30(1):61-86.

7. Freud S. Three Contributions to the Theory of Sex. New York, NY: E.P. Dutton; 1962.

8. Fast I. Aspects of core gender identity. Psychoanal Dial. 1999;9(5): 633-661.

9. Stoller R. Presentations of Gender. New Haven, CT: Yale University Press; 1985.

10. Ehrensaft D. Gender Born, Gender Made. New York, NY: The Experiment Press; 2011.

11. Diamond M. Sex and gender: same or different? Feminism Psychol. 2000;10(1):46-54.

12. Vanderhorst $B$. Whither lies the self: intersex and transgender individuals and a proposal for brain-based legal sex. Harvard Law \& Policy Review. 2015;9:241-274.

13. Ehrensaft D. From gender identity disorder to gender identity creativity: true gender self child therapy. J Homosex. 2012;59(3):337-356.

14. Sherer I, Rosenthal S, Ehrensaft D, Baum J. Child and adolescent gender center: a multidisciplinary collaboration to improve the lives of gender nonconforming children and teens. Pediatr Rev. 2012;33(6): 273-276.

15. Green R. The 'Sissy boy'Syndrome and the Development of Homosexuality. New Haven, CT: Yale University Press; 1987:295.

16. Zucker K, Bradley S. Gender Identity Disorder and Psychosexual Problems in Children and Adolescents. New York, NY: The Guilford Press; 1995.

17. Cross JM. Outcry prompts CAMH to review its controversial treatment of trans youth. Metro News, Toronto; 2015, March 18; online. Available from: http://metronews.ca/news/toronto/1315743/outcry-promptscamh-to-review-its-controversial-treatment-of-trans-youth

18. Cohen-Kettenis PT, Pfäfflin F. Transgenderism and Intersexuality in Childhood and Adolescence. Thousand Oaks, CA: Sage Publications; 2003.

19. Steensma TD, McGuire J, Kreukels BP, Beekman AJ, Cohen-Kettenis PT. Factors associated with desistence and persistence of childhood gender dysphoria: a quantitative follow-up study. $J$ Am Acad Child Adolesc Psychiatry. 2013;52(6):582-590. 
20. Steensma TD, Biemond R, de Boer F, Cohen-Kettenis PT. Desisting and persisting gender dysphoria after childhood: a qualitative follow-up study. Clin Child Psychol Psychiatry. 2011;16(4):499-516.

21. Ehrensaft D. The Gender Creative Child. New York, NY: The Experiment; 2016.

22. Ehrensaft D. Boys will be girls, girls will be boys. Psychoanalytic Psychol. 2011;28(4):528-548.

23. Ehrensaft D. From gender Identity disorder to gender identity creativity: true gender self child therapy. J Homosex. 2012;59(3):337-356.

24. Malpas J. Between pink and blue: a multi-dimensional family approach to gender non-conforming children and their families. Fam Process. 2011;50(4):453-470.

25. Roberts L, Rosario M, Corliss HL, et al. Childhood gender nonconformity: a risk indicator for childhood abuse and post-traumatic stress in youth. Pediatrics. 2012;129:410-417.

26. Wallace R, Russell H. Attachment and shame in gender-nonconforming children and their families: toward a theoretical framework for evaluating clinical interventions. Int J Transgenderism. 2013;14(3):113-126.
27. Olson KR, Key AC, Eaton NR. Gender cognition in transgender children. Psychol Sci. 2015;26(4):467-474.

28. Durwood L, McLaughlin KA, Olson KR. Mental health and self-worth in socially transitioned transgender youth. J Am Acad Child Adolesc Psychiatry. 2017;56(2):116-123.

29. Rosenthal SM. Approach to the patient: transgender youth: endocrine considerations. J Clin Endocrinol Metab. 2014;99(12):4379-4389.

30. Vance SR, Ehrensaft D, Rosenthal SM. Psychological and medical care of gender nonconforming youth. Pediatrics. 2014;134(6):1184-1192.

31. Ehrensaft D, Hastings J, Hsaio K. Ferility issues for youth: puberty blockers, hormones. Decision-making through the lens of family building options. World Professional Association for Transgender Health Symposia. Amsterdam, June 20, 2016.

32. Harris A. Gender as Soft Assembly. New York, NY: The Analytic Press; 2005.

33. deVries ALC, McGuire JK, Steensma TD, Wagenaar EC, Doreleijers TA, Cohen-Kettenis PT. Young adult psychological outcome after puberty suppression and gender reassignment. Pediatrics. 2014;134(4):696-704
Adolescent Health, Medicine and Therapeutics

\section{Publish your work in this journal}

Adolescent Health, Medicine and Therapeutics is an international, peer-reviewed, open access journal focusing on health, pathology, and treatment issues specific to the adolescent age group. All aspects of health maintenance, preventative measures and disease treatment interventions are addressed within the journal and practitioners from all disciplines are

\section{Dovepress}

invited to submit their work as well as healthcare researchers and patient support groups. This journal is included in PubMed. The manuscript management system is completely online and includes a very quick and fair peer-review system. Visit http://www.dovepress.com/testimonials. php to read real quotes from published authors. 